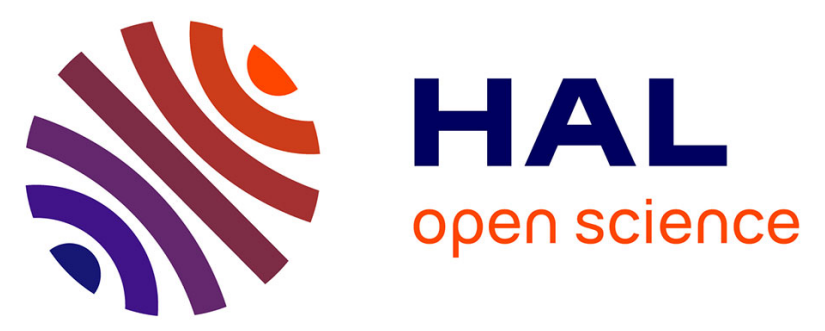

\title{
Multireflection grazing-incidence X-ray diffraction: A new approach to experimental data analysis
}

Marianna Marciszko-Wiąckowska, Adrian Oponowicz, Andrzej Baczmanski, Miroslaw X. Wróbel, Chedly Braham, Roman Wawszczak

\section{- To cite this version:}

Marianna Marciszko-Wiąckowska, Adrian Oponowicz, Andrzej Baczmanski, Miroslaw X. Wróbel, Chedly Braham, et al.. Multireflection grazing-incidence X-ray diffraction: A new approach to experimental data analysis. Journal of Applied Crystallography, 2019, 52, pp.1409-1421. 10.1107/S1600576719013876 . hal-02456399

\section{HAL Id: hal-02456399 \\ https://hal.science/hal-02456399}

Submitted on 27 Jan 2020

HAL is a multi-disciplinary open access archive for the deposit and dissemination of scientific research documents, whether they are published or not. The documents may come from teaching and research institutions in France or abroad, or from public or private research centers.
L'archive ouverte pluridisciplinaire HAL, est destinée au dépôt et à la diffusion de documents scientifiques de niveau recherche, publiés ou non, émanant des établissements d'enseignement et de recherche français ou étrangers, des laboratoires publics ou privés. 
Keywords: residual stress; multireflection grazing-incidence X-ray diffraction; coating layers; elastic constants.
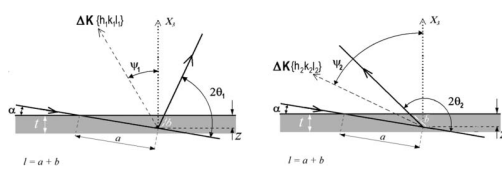

\section{Multireflection grazing-incidence X-ray diffraction: a new approach to experimental data analysis}

\author{
Marianna Marciszko-Wiąckowska, ${ }^{\mathrm{a} *}$ Adrian Oponowicz, ${ }^{\mathrm{b}}$ Andrzej Baczmański, \\ Miroslaw Wróbel, ${ }^{\mathrm{c}}$ Ch. Braham ${ }^{\mathrm{d}}$ and R. Wawszczak ${ }^{\mathrm{b}}$
}

\begin{abstract}
aGH University of Science and Technology, Academic Centre for Materials and Nanotechnology, aleja A. Mickiewicza 30, 30-059 Krakow, Poland, ${ }^{\mathbf{b}} \mathrm{AGH}$ University of Science and Technology, Faculty of Physics and Applied Computer Science, aleja A. Mickiewicza 30, 30-059 Krakow, Poland, ' AGH University of Science and Technology, Faculty of Metals Engineering and Industrial Computer Science, aleja A. Mickiewicza 30, 30-059 Krakow, Poland, and ${ }^{\mathbf{d}}$ Arts et Métiers-ParisTech, PIMM, CNRS UMR 8006, 151 Boulevard de I'Hôpital, 75013 Paris, France. *Correspondence e-mail: marciszk@agh.edu.pl
\end{abstract}

The multireflection grazing-incidence X-ray diffraction method is used to test surface stresses at depths of several micrometres in the case of metal samples. This work presents new ways of analysing experimental data obtained by this method for Ni samples exhibiting significant elastic anisotropy of crystals. Three different methods of determining biaxial stresses and lattice parameter were compared. In the first approach, the calculations were performed using the linear least-squares method, and then two simplified procedures based on simple linear regression (weighted and non-weighted) were applied. It was found that all the tested methods give similar results, i.e. almost equal values of the determined stresses and lattice parameters and the uncertainties of their determination. The advantage of analyses based on simple linear regression is their simplicity and straightforward interpretation, enabling easy verification of the influence of the crystallographic texture and the presence of shear stresses, as well as graphical determination of the stress-free lattice parameter.

\section{Introduction}

The well known methodology of stress determination in polycrystalline materials is based on diffraction measurements of lattice strains. Different techniques have been developed over almost one hundred years of using this method, but the most widespread and simplest one is based on X-ray measurement of the $\sin ^{2} \psi$ plot, in which simple linear regression is used (Hauk, 1997; Noyan \& Cohen, 1987). This method allows the determination of not only the components of the stress tensor but also the stress-free lattice parameter (assuming stress normal to the surface equal to zero).

The idea of stress analysis using diffraction is based on the measurement of crystallographic lattice strains caused by residual stresses of different types. The first- and second-order stresses cause mean elastic lattice strains for groups of polycrystalline grains. This effect can be seen as a shift of the diffraction peaks. In order to determine stresses, first the diffraction peaks are measured at different orientations of the scattering vector with respect to the sample (Fig. 1). The peaks' positions are found by fitting theoretical functions (e.g. the pseudo-Voigt profile; Thompson et al., 1987) to the experimental data. Then, using Bragg's relation the mean lattice strain can be determined as the relative change of interplanar spacing, i.e. 


$$
\begin{aligned}
\langle\varepsilon(\phi, \psi)\rangle_{\{h k l\}} & =-\cot \theta_{\{h k l\}}^{0}\left(\langle\theta\rangle_{\{h k l\}}-\theta_{\{h k l\}}^{0}\right) \text { or } \\
\langle\varepsilon(\phi, \psi)\rangle_{\{h k l\}} & =\frac{\langle d\rangle_{\{h k l\}}-d_{\{h k l\}}^{0}}{d_{\{h k l\}}^{0}}
\end{aligned}
$$

where $\langle\ldots\rangle_{\{h k l\}}$ denotes a mean value defined for the volume of the crystallites that take part in diffraction, and $\langle d\rangle_{\{h k l\}}$ and $d_{\{h k l\}}^{0}$ are the mean interplanar spacings for the $\{h k l\}$ crystallographic planes determined in the studied sample and in a stress-free crystallite, respectively. The corresponding shift of the diffraction peak is equal to $2\left(\langle\theta\rangle_{\{h k l\}}-\theta_{\{h k l\}}^{0}\right)$, where $2 \theta$ is the diffraction angle defined as the angle between the incident and the diffracted X-ray beam.

In general, the relation between the strains $\langle\varepsilon(\phi, \psi)\rangle_{\{h k l\}}$ measured using the $h k l$ reflection in the direction of the diffraction vector $\Delta \mathbf{K}$ (defined by the $\phi$ and $\psi$ angles in Fig. 1) and stress tensor $\sigma_{i j}$ defined in the sample reference frame is given by the equation (Hauk, 1997; Noyan \& Cohen, 1987)

$$
\langle\varepsilon(\phi, \psi)\rangle_{\{h k l\}}=F_{i j}(h k l, \phi, \psi, f) \sigma_{i j}
$$

where $F_{i j}$ are the X-ray stress factors (XSFs) depending not only on the $h k l$ reflection but also on crystallographic texture, $f$ represents the orientation distribution function (ODF) and the sum is calculated for six independent components of the stress tensor (Barral et al., 1987; Van Houtte \& De Buyser, 1993; Hauk, 1997; van Leeuwen et al., 1999; Genzel, 1999; Leoni et al., 2001; Welzel \& Mittemeijer, 2003; Welzel et al., 2005).

When using diffraction methods, the lattice strains are not measured directly but in fact the interplanar spacings $\langle d(\phi, \psi)\rangle_{\{h k l\}}$ are determined from the diffraction peak positions, measured for different orientations of the scattering vector. Therefore, the above equation can be rewritten in the following form $[c f$. equations (1) and (2)]:

$$
\langle d(\phi, \psi)\rangle_{\{h k l\}}=F_{i j}(h k l, \phi, \psi, f) \sigma_{i j} d_{h k l}^{0}+d_{h k l}^{0} .
$$

Finally, equation (3) is used to determine the components $\sigma_{i j}$ of the stress tensor and the stress-free spacing $d_{h k l}^{0}$ (if it is unknown), which are adjusted in the least-squares procedure in order to fit the calculated $\langle d(\phi, \psi)\rangle_{\{h k l\}}$ values to the experimental ones. The procedure is based on minimizing the merit function called $\chi^{2}$, which is defined as

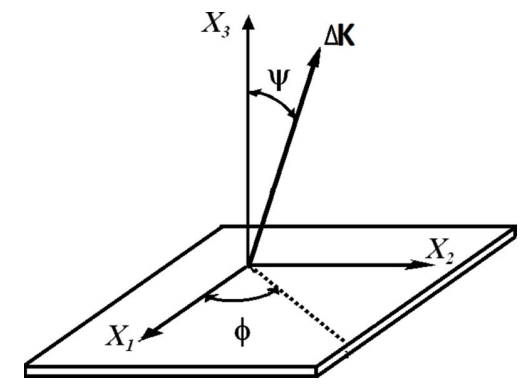

Figure 1

Orientation of the scattering vector $\Delta \mathbf{K}$ with respect to the sample system $\mathbf{X}$, and definition of the $\psi$ and $\phi$ angles.

$$
\chi^{2}=\frac{1}{N-M} \sum_{n=1}^{N}\left[\frac{\left\langle d\left(\phi_{n}, \psi_{n}\right)\right\rangle_{\{h k l\}}^{\exp }-\left\langle d\left(\phi_{n}, \psi_{n}\right)\right\rangle_{\{h k l\}}^{\mathrm{cal}}}{\delta_{n}}\right]^{2},
$$

where $\left\langle d\left(\phi_{n}, \psi_{n}\right)\right\rangle_{\{h k l\}}^{\exp }$ and $\left\langle d\left(\phi_{n}, \psi_{n}\right)\right\rangle_{\{h k l\}}^{\text {cal }}$ are, respectively, the experimental and calculated lattice parameters, $\delta_{n}=$ $\delta\left[\left\langle d\left(\phi_{n}, \psi_{n}\right)\right\rangle_{\{h k l\}}^{\exp }\right]$ is the measurement uncertainty (i.e. standard deviation) of $\left\langle d\left(\phi_{n}, \psi_{n}\right)\right\rangle_{\{h k l\}}^{\exp }$ for the $n$th measurement, and $N$ and $M$ are the number of measured points and fitting parameters, respectively. If the uncertainties $\delta_{n}$ are known, the value of $\chi^{2}$ is a measure of the goodness of fit, i.e. $\chi^{2}=1$ means that a 'good fit', corresponding to experimental uncertainty, was obtained (Press et al., 1992).

In the case of quasi-isotropic (non-textured) polycrystalline materials, $F_{i j}(h k l, \phi, \psi, f)$ can be expressed through two independent X-ray elastic constants (XECs) $s_{1}^{h k l}$ and $s_{2}^{h k l}$ (Hauk, 1997; Noyan \& Cohen, 1987):

$$
\begin{aligned}
F_{11}(h k l, \phi, \psi, f) & =\frac{1}{2} s_{2}^{h k l} \cos ^{2} \phi \sin ^{2} \psi+s_{1}^{h k l}, \\
F_{22}(h k l, \phi, \psi, f) & =\frac{1}{2} s_{2}^{h k l} \sin ^{2} \phi \sin ^{2} \psi+s_{1}^{h k l}, \\
F_{33}(h k l, \psi, f) & =\frac{1}{2} s_{2}^{h k l} \cos ^{2} \psi+s_{1}^{h k l}, \\
F_{12}(h k l, \phi, \psi, f) & =\frac{1}{2} s_{2}^{h k l} \sin 2 \phi \sin ^{2} \psi, \\
F_{13}(h k l, \phi, \psi, f) & =\frac{1}{2} s_{2}^{h k l} \cos \phi \sin 2 \psi, \\
F_{23}(h k l, \phi, \psi, f) & =\frac{1}{2} s_{2}^{h k l} \sin \phi \sin 2 \psi .
\end{aligned}
$$

The values of the XSFs or XECs can be evaluated experimentally by applying known external loads to the sample and measuring the lattice strains for different orientations of the scattering vector. Alternatively, the XSFs or XECs can be calculated from single-crystal elastic constants (SECs) using a chosen model of crystallite interaction (Dölle, 1979; Dölle \& Cohen, 1980; Barral et al., 1987; Brakman, 1987; Baczmański et al., 1993, 2003; Matthies et al., 1994; Welzel et al., 2005). The Voigt (1928) and Reuss (1929) methods for calculation of the XECs or XSFs are based on the hypothesis of strain or stress homogeneity in the considered volume, respectively, and this assumption can be applied to quasi-isotropic and textured polycrystalline materials. However, in order to account for the interaction between grains in the calculations, it is necessary to use, for example, the Eshelby-Kröner method (Kröner, 1961), in which the grains are approximated by ellipsoidal inclusions (Eshelby, 1957) embedded in a homogeneous medium. In addition, to take into account the direction-dependent interaction between grains, two approaches can be used. The first was given by Vook \& Witt (1965) and developed by van Leeuwen et al. (1999) and Welzel \& Mittemeijer (2003) for columnar grains in the surface layer. In this approach, grains having dimensions equal to the thickness of the film exhibit the same in-plane strain (a Voigt-type behaviour), whereas they can deform freely in the direction perpendicular to the surface (a Reuss-type behaviour). The second approach, called the free-surface model, was proposed by Baczmanski et al. (2008) for grains placed close to the sample surface. Similarly as in the Vook-Witt model, it was assumed that grains can freely deform in the direction normal to the surface (Reuss-type behaviour), while the in-plane interaction is 
approximated by the self-consistent model (Eshelby-Krönertype behaviour). It should be highlighted that the latter approximation describes the interaction occurring between the grains in the gauge volume penetrated by X-rays near the surface.

After simple transformation of equation (3) and using equation (5) for quasi-isotropic material, the interplanar spacings can be expressed by the macrostresses $\sigma_{i j}$ and the stress-free interplanar spacing $d_{\{h k l\}}^{0}$ :

$$
\begin{aligned}
& \langle d(\phi, \psi)\rangle_{\{h k l\}}=\left\{\frac { 1 } { 2 } s _ { 2 } ^ { h k l } \left[\left(\sigma_{11}-\sigma_{33}\right) \cos ^{2} \phi\right.\right. \\
& \left.\quad+\sigma_{12} \sin 2 \phi+\left(\sigma_{22}-\sigma_{33}\right) \sin ^{2} \phi\right] \sin ^{2} \\
& \quad+s_{1}^{h k l}\left(\sigma_{11}+\sigma_{22}+\sigma_{33}\right)+\frac{1}{2} s_{2}^{h k l} \sigma_{33} \\
& \left.\quad+\frac{1}{2} s_{2}^{h k l}\left(\sigma_{13} \cos \phi+\sigma_{23} \sin \phi\right) \sin 2 \psi\right\} d_{h k l}^{0}+d_{h k l}^{0}
\end{aligned}
$$

When one $h k l$ reflection is used in the experiment, equation (6) leads to the well known $\sin ^{2} \psi$ law, in which the measured interplanar spacings are plotted versus $\sin ^{2} \psi$, for constant $\phi$ angle (Hauk, 1997). In the case of zero values of shear stresses, the plot of $\langle d(\phi, \psi)\rangle_{\{h k l\}}$ versus $\sin ^{2} \psi$ is linear and the normal stresses $\sigma_{11}, \sigma_{22}$ and $\sigma_{33}$ can be determined from a simple linear regression (i.e. by fitting a straight line to the experimental data), if $d_{h k l}^{0}$ is known. Alternatively, in the case of X-ray diffraction, when forces normal to the surface can be neglected owing to the shallow information depth (i.e. $\sigma_{33}=0$ ), the values of $\sigma_{11}, \sigma_{22}$ and $d_{h k l}^{0}$ can be found. This is the principle of the simplest methodology for stress measurement, which is widely used in the community and fully supported by the available commercial software. However, many specific methodologies for stress measurement have been proposed, and they require simple and clear interpretations. One such method is multireflection grazing-incidence X-ray diffraction (MGIXD), developed by Marciszko et al. $(2013,2016)$ and used for the determination of stress variation under the sample surface. In this work a new simple method for interpretation of MGIXD measurements is proposed and tested. The main advantage of this method is its simplicity and the possibility of clear results presentation. vector (variable $2 \theta_{\{h k l\}}$ angle for a constant wavelength; see Fig. 2) given by the equation

$$
\psi_{h k l}=\theta_{\{h k l\}}-\alpha,
$$

where $2 \theta_{\{h k l\}}$ are the diffraction angles corresponding to those reflections $h k l$ for which diffraction peaks are measured (Skrzypek et al., 2001; Marciszko et al., 2012, 2013, 2016).

If the incident angle $\alpha$ is low and constant during the measurement, the penetration depth of the X-rays depends mostly on the long path of the incident beam in the studied material ( $a \gg b$ in Fig. 2) and it does not depend significantly on the $2 \theta_{\{h k l\}}$ angle. Therefore the information/penetration depth for a given incident angle $\alpha$ can be calculated from the formula

$$
\tau=\left[\frac{\mu}{\sin \alpha}+\frac{\mu}{\sin \left(\theta_{\{h k l\}}-\alpha\right)}\right]^{-1} \simeq \frac{\sin \alpha}{\mu},
$$

where $\mu$ is the linear attenuation coefficient for X-ray radiation.

The stress is determined for a constant penetration depth (constant incident angle $\alpha$ ) by measuring interplanar spacings for different $h k l$ reflections corresponding to different $\psi_{h k l}$ angles (Fig. 1). Subsequently, the incident angle can be changed in order to perform measurement for another penetration depth. The advised experimental configuration for this experiment is the parallel-beam geometry, minimizing possible error connected with positioning of the sample, which can significantly affect the value of determined stress. For more details see Marciszko et al. (2015, 2016).

To determine the components of the stress tensor and strain-free lattice parameter, equation (3) should be modified in order to relate the lattice strains to the constant reference value ( $a_{0}$ lattice parameter) instead of the reflectiondependent $d_{h k l}^{0}$ spacings. Therefore the equivalent lattice parameters $\langle a(\phi, \psi)\rangle_{\{h k l\}}$ are calculated and together with the $a_{0}$ lattice parameter are introduced into equation (3) (Hauk, 1997; Genzel, 1999; Skrzypek et al., 2001; Welzel \& Mittemeijer, 2003; Welzel et al., 2005; Marciszko et al., 2015):

$$
\langle a(\phi, \psi)\rangle_{\{h k l\}}=F_{i j}(h k l, \phi, \psi, f) \sigma_{i j} a_{0}+a_{0},
$$

where for the cubic crystal structure

\section{Stress measurements using the MGIXD method}

\subsection{Experimental principles}

The MGIXD geometry (Skrzypek et al., 2001) is based on the idea of the low-incident-beam-angle diffraction method (Van Acker et al., 1994). It has been developed in order to study the depth profile of residual stresses in near-surface layers of a polycrystalline material. The used geometry is characterized by a small and constant incident angle $\alpha$, in omega acquisition mode (Welzel et al., 2005), and by different orientations of the scattering
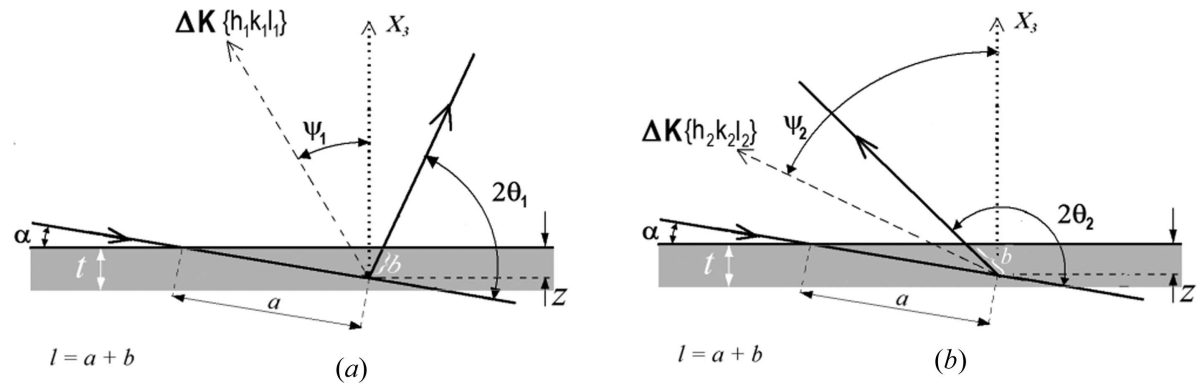

Figure 2

Geometry of the MGIXD method. The incident angle $\alpha$ is fixed during measurement, while the orientation of the scattering vector is characterized by the $\psi_{h k l}$ angle (between the scattering vector $\Delta \mathbf{K}$ and the normal to the sample $\mathbf{X}_{3}$ ). The path of the X-rays in the material is shown (path length $l=$ $a+b$, where $a \gg b$ ). 


$$
\langle a(\phi, \psi)\rangle_{\{h k l\}}=\langle d(\phi, \psi)\rangle_{\{h k l\}}\left(h^{2}+k^{2}+l^{2}\right)^{1 / 2}
$$

and $\phi$ can be chosen arbitrarily, while $\psi$ depends on the diffraction angle for the given reflection $h \mathrm{kl}$.

Until now the analysis of the experimental data was based on the linear least-squares method, in which the $\sigma_{i j} a_{0}$ and $a_{0}$ linear parameters of equation (9) are adjusted in order to fit the calculated $\langle a(\phi, \psi)\rangle_{\{h k l\}}$ values to the experimental ones. With this aim, the merit function given by equation (4), in which interplanar spacings $\langle d(\phi, \psi)\rangle_{\{h k l\}}$ are replaced by the equivalent lattice parameters $\langle a(\phi, \psi)\rangle_{\{h k l\}}$, is minimized:

$$
\chi^{2}=\frac{1}{N-M} \sum_{n=1}^{N}\left[\frac{\left\langle a\left(\phi_{n}, \psi_{n}\right)\right\rangle_{\{h k l\}}^{\exp }-\left\langle a\left(\phi_{n}, \psi_{n}\right)\right\rangle_{\{h k l\}}^{\mathrm{cal}}}{\delta_{n}}\right]^{2},
$$

where $\delta_{n}=\delta\left[\left\langle a\left(\phi_{n}, \psi_{n}\right)\right\rangle_{\{h k l\}}^{\exp }\right], M=3$ is the number of fitted parameters and $N$ is the number of measured points.

As a result, the $a_{0}$ stress-free lattice parameters as well as the stress components $\sigma_{i j}$ can be determined. We emphasize that equation ( $9 a)$ is written for cubic crystals, while analogous formulas for orthorhombic or hexagonal crystals contain also a $c / a$ parameter which in principle is unknown. This leads to more complex iterative data treatment which was proposed elsewhere (e.g. Marciszko et al., 2016).

The advantage of the linear least-squares method applied in the present work [based on equation (9)] is that the obtained solution is unique and corresponds to the maximum likelihood of the obtained result. According to the Gauss-Markov theorem, the estimators of the determined parameters are unbiased and exhibit the lowest variance (known as the best linear unbiased estimator). The uncertainties of the determined fitting parameters can be calculated using the rule of error propagation, if the uncertainties of the measured lattice parameters $\delta\left[\left\langle a\left(\phi_{n}, \psi_{n}\right)\right\rangle_{\{h k l\}}^{\exp }\right]$ are known. However, the uncertainties of the measured peak positions [and consequently of $\left.\langle a(\phi, \psi)\rangle_{\{h k l\}}\right]$ are not easy to determine, and they are usually larger than those obtained directly from the diffraction peak adjustment (especially for low $2 \theta_{\{h k l\}}$ angle reflections). Therefore, it is more reasonable to calculate the statistical uncertainties of the adjusted parameters, resulting from the values of residuals [i.e. differences between measured and fitted $\langle a(\phi, \psi)\rangle_{\{h k l\}}$ values] with the assumption that a good fit was reached (Hauk, 1997). The problem of experimental uncertainties will be discussed in the next sections. For details concerning the linear least-squares calculations and uncertainty analysis used in this work, see the singular value decomposition procedure described by Press et al. (1992).

The results of the analysis are presented as an $\langle a(\phi, \psi)\rangle_{\{h k l\}}$ versus $\sin ^{2} \psi$ plot (cf. Fig. 5 below), similarly as in the standard method in which the measurements are performed for one $h \mathrm{kl}$ reflection. However, the plot obtained for different $h \mathrm{kl}$ reflections is not linear versus $\sin ^{2} \psi$ owing to anisotropy of the SECs, which leads to different values of XECs for different $h k l$ reflections. Furthermore, crystallographic texture influences $F_{i j}(h k l, \phi, \psi, f)$ and can cause additional nonlinearities of the $\langle a(\phi, \psi)\rangle_{\{h k l\}}$ versus $\sin ^{2} \psi$ plot.

\subsection{Concepts of experimental data analysis}

In this work three ways of determining the biaxial stress and $a_{0}$ parameter are presented. The first procedure is the most general one and can be used for samples with any type of crystallographic texture, while the two new methods proposed in the present paper are far more simple but in principle they can be applied in the case of fibre texture or for quasi-isotropic (i.e. non-textured) materials, on the assumption that the shear stresses can be neglected. It will be shown that the latter methods also give reasonable results if the texture is weak.

2.2.1. Weighted linear least-squares method. In the first approach, calculations are performed using the weighted linear least-squares (WLLS) method based on equation (9). In this case the fitting parameters $\sigma_{11} a_{0}$ and $\sigma_{22} a_{0}$ as well as the value of $a_{0}$ are adjusted (on the assumption that $\sigma_{33}=0$ ), and then the biaxial stress components $\sigma_{11}$ and $\sigma_{22}$ are computed together with their uncertainties. In principle this analysis can be applied in the case of nonzero shear stresses, but in this work it is assumed that these stresses are neglectable. The values of $F_{i j}(h k l, \phi, \psi, f)$ used in equation (9) are calculated from SECs using the ODF as the weighting function, if crystallographic texture is significant (Baczmański et al., 2003, 2008).

As mentioned above, an important issue when determining the stresses is the uncertainty analysis and the appropriate definition of weights $1 / \delta_{n}^{2}$ in the calculation of the merit function given by equation (10). Our previous experiments performed with the sample under external loading (Marciszko et al., 2015, 2016) as well as for powder samples show that the uncertainties of peak positions given by the fitting procedure (with a pseudo-Voigt function) are underestimated in comparison with residuals given by the least-squares fitting, especially in the case of low $2 \theta_{\{h k l\}}$ angle reflections. This is caused by instrumental errors, the influence of microstresses, stress heterogeneity and many unknown factors which are difficult to estimate. Because in MGIXD low $2 \theta_{\{h k l\}}$ angle reflections are necessary in order to increase the range of $\sin ^{2} \psi$, a method of data analysis that takes into account the different weights of the measured points was proposed and tested, in order to estimate the statistical uncertainties of the determined stresses and $a_{0}$ lattice parameter. In calculations, a constant uncertainty $\delta\left(2 \theta_{\{h k l\}}\right)=0.01^{\circ}$ for the determined peak positions $2 \theta_{\{h k l\}}$ was assumed, and the corresponding uncertainties of $\langle a(\phi, \psi)\rangle_{\{h k l]}$ were calculated from the equation

$$
\delta\left[\langle a(\phi, \psi)\rangle_{\{h k l]}^{\exp }\right]=\left(h^{2}+k^{2}+l^{2}\right)^{1 / 2} \cot \theta_{\{h k l\}} \delta_{2 \theta_{[h k l]}} .
$$

Note that, owing to the $\cot \theta_{\{h k l\}}$ multiplier in equation (11), the uncertainties $\delta\left[\langle a(\phi, \psi)\rangle_{\{h k l\}}^{\exp }\right]$ are much lower for higher $2 \theta_{\{h k l\}}$ angles. Because the inverse of squared uncertainties plays the role of a weight in the merit function [equation (10)], the values of $\langle a(\phi, \psi)\rangle_{\{h k l\}}$ measured with higher $2 \theta_{\{h k l\}}$ angles have a much greater impact on the finally determined stresses and $a_{0}$ lattice parameter, as compared with those measured with lower $2 \theta_{\{h k l\}}$ angles. As already mentioned, the results of this method of data analysis are presented as $\langle a(\phi, \psi)\rangle_{\{h k l\}}$ 
versus $\sin ^{2} \psi$ plots, which are not linear and are difficult to interpret in a straightforward manner.

The statistical uncertainties, calculated in this work with the singular value decomposition procedure described by Press $e t$ al. (1992), do not depend on the arbitrarily chosen value of $\delta\left(2 \theta_{\{h k l]}\right)$ and only the relations between uncertainties [given by equation (11)] are important. On the other hand our previous tests showed that the assumed value $\delta\left(2 \theta_{\{h k l\}}\right)=0.01^{\circ}$ gives reasonable values of the merit function $\chi^{2}$, and the resulting relation with $\delta\left[\langle a(\phi, \psi)\rangle_{\{h k l\}}^{\exp }\right]$ given by equation (11) approximately mimics the length of the error bar compared with the residuals (Marciszko et al., 2015, 2016). In addition, this assumption was tested previously by Hauk (1997). Note also that if the $\delta\left(2 \theta_{\{h k l]}\right)$ value is assumed arbitrarily the merit function $\chi^{2}$ given by equation (10) can be calculated, but it cannot be treated as the goodness-of-fit indicator. However, the $\chi^{2}$ value still can be used to compare the quality of fitting, e.g. for different models of XSFs (or XECs) used in stress analysis.

2.2.2. Weighted and simple linear regression. In the second approach a simple linear regression is proposed to determine biaxial stresses and lattice parameter from experimental data obtained using the MGIXD method. In the present paper, the name 'simple linear regression' is reserved for the linear leastsquares procedure in which a straight line is fitted to experimental points. The proposed method can be applied with the assumption of a quasi-isotropic material or fibre type of texture. The shear stresses are not considered in this analysis; if they are significant they will cause nonlinearities of the $\langle a(\phi, \psi)\rangle_{\{h k l\}}$ versus $\sin ^{2} \psi$ plots. Assuming a quasi-isotropic material or fibre type of texture (axially symmetrical with respect to the axis $X_{3}$, defined in Fig. 1), we can write

$$
\begin{aligned}
& F_{11}\left(h k l, \phi=0^{\circ}, \psi, f\right)=F_{22}\left(h k l, \phi=90^{\circ}, \psi, f\right)=G(h k l, \psi), \\
& F_{22}\left(h k l, \phi=0^{\circ}, \psi, f\right)=F_{11}\left(h k l, \phi=90^{\circ}, \psi, f\right)=H(h k l, \psi),
\end{aligned}
$$

where $G(h k l, \psi)$ and $H(h k l, \psi)$ depend on the $h k l$ reflection and $\psi$ angle but not on the $\phi$ angle (for axial symmetry about the $X_{3}$ axis).

Neglecting insignificant shear stresses and taking into account equation (12), we can write equation (9) for measurements performed for two values of $\phi$ angle, i.e. $\phi=0^{\circ}$ and $\phi=90^{\circ}$ :

$$
\begin{aligned}
\left\langle a\left(\phi=0^{\circ}, \psi\right)\right\rangle_{\{h k l\}} & =\left[G(h k l, \psi) \sigma_{11}+H(h k l, \psi) \sigma_{22}\right] a_{0}+a_{0}, \\
\left\langle a\left(\phi=90^{\circ}, \psi\right)\right\rangle_{\{h k l\}} & =\left[H(h k l, \psi) \sigma_{11}+G(h k l, \psi) \sigma_{22}\right] a_{0}+a_{0} .
\end{aligned}
$$

The sum and difference of the above equations are equal to

$$
\begin{aligned}
& \frac{\left\langle a\left(\phi=0^{\circ}, \psi\right)\right\rangle_{\{h k l\}}+\left\langle a\left(\phi=90^{\circ}, \psi\right)\right\rangle_{\{h k l\}}}{2} \\
& \quad=\frac{G(h k l, \psi)+H(h k l, \psi)}{2}\left(\sigma_{11}+\sigma_{22}\right) a_{0}+a_{0}, \\
& \left\langle a\left(\phi=0^{\circ}, \psi\right)\right\rangle_{\{h k l\}}-\left\langle a\left(\phi=90^{\circ}, \psi\right)\right\rangle_{\{h k l\}} \\
& \quad=[G(h k l, \psi)-H(h k l, \psi)]\left(\sigma_{11}-\sigma_{22}\right) a_{0},
\end{aligned}
$$

or in the case of the quasi-isotropic sample

$$
\begin{aligned}
& \frac{\left\langle a\left(\phi=0^{\circ}, \psi\right)\right\rangle_{\{h k l\}}+\left\langle a\left(\phi=90^{\circ}, \psi\right)\right\rangle_{\{h k l\}}}{2} \\
& \quad=\left(\frac{1}{4} s_{2}^{h k l} \sin ^{2} \psi+s_{1}^{h k l}\right)\left(\sigma_{11}+\sigma_{22}\right) a_{0}+a_{0}, \\
& \left\langle a\left(\phi=0^{\circ}, \psi\right)\right\rangle_{\{h k l\}}-\left\langle a\left(\phi=90^{\circ}, \psi\right)\right\rangle_{\{h k l\}} \\
& \quad=\left(\frac{1}{2} s_{2}^{h k l} \sin ^{2} \psi\right)\left(\sigma_{11}-\sigma_{22}\right) a_{0} .
\end{aligned}
$$

Both sets of equations (14) and (15) can be rewritten in the linear form

$$
y=m x+a_{0}, \quad y^{\prime}=m^{\prime} x^{\prime},
$$

where $y=\left[\left\langle a\left(\phi=0^{\circ}, \psi\right)\right\rangle_{\{h k l\}}+\left\langle a\left(\phi=90^{\circ}, \psi\right)\right\rangle_{\{h k l]}\right] / 2, \quad x=$ $[G(h k l, \psi)+H(h k l, \psi)] / 2$ (or for a quasi-isotropic sample $x=$ $\left.\frac{1}{4} s_{2}^{h k l} \sin ^{2} \psi+s_{1}^{h k l}\right), m=\left(\sigma_{11}+\sigma_{22}\right) a_{0}, y^{\prime}=\left\langle a\left(\phi=0^{\circ}, \psi\right)\right\rangle_{\{h k l\}}-$ $\left\langle a\left(\phi=90^{\circ}, \psi\right)\right\rangle_{\{h k l\}}, x^{\prime}=[G(h k l, \psi)-H(h k l, \psi)]$ (or for a quasi-isotropic sample $\left.x^{\prime}=\frac{1}{2} s_{2}^{h k l} \sin ^{2} \psi\right)$ and $m^{\prime}=\left(\sigma_{11}-\right.$ $\left.\sigma_{22}\right) a_{0}$.

Note that the above functions are linear versus the sum or differences of XSFs (in the case of a quasi-isotropic sample expressed by $s_{2}^{h k l}, s_{1}^{h k l}$ and $\psi$ [cf. equation (15)], while the parameters of these functions $\left(m, m^{\prime}\right.$ and $\left.a_{0}\right)$ are expressed by the stress components and stress-free lattice parameter. It should be emphasized that the presented 'linearization' of the function used in stress analysis is in principle similar to the ideas of Dölle \& Hauk (1976) and Dölle (1979), proposed to compensate the nonlinearity caused by shear stresses. In the case of Dölle's method, instead of ellipse fitting to the $\langle d(\phi, \psi)\rangle_{\{h k l\}}$ versus $\sin ^{2} \psi$ plots (split for $\phi$ and $\phi+180^{\circ}$ angles), two linear least-squares regressions were proposed for the $\langle d(\phi, \psi)\rangle_{\{h k l\}}+\left\langle d\left(\phi+180^{\circ}, \psi\right)\right\rangle_{\{h k l\}}$ versus $\sin ^{2} \psi$ and $\langle d(\phi, \psi)\rangle_{\{h k l\}}-\left\langle d\left(\phi+180^{\circ}, \psi\right)\right\rangle_{\{h k l\}}$ versus $\sin ^{2} \psi$ linear functions. As a result, the components of the stress tensor, including shear stresses, could be determined. In the present work, the effect of crystal elastic anisotropy in the multireflection method is compensated for by calculating the sum or the difference of lattice parameters measured at $\phi=0^{\circ}$ and $\phi+$ $90^{\circ}$ angles. In this case the resulting functions are linear versus the sum or difference of the appropriate XSF (or XEC) values [cf. equations (14), (15) and (16)].

Applying this simple linear regression procedure, the parameters of both equations (16) can be found. The value of the stress-free lattice parameter $a_{0}$ can be directly determined from the first of equations (16) as the intercept of this function with the $y$ axis. The slopes of the first and the second plots described by equation (16) determine, respectively, the sum of the stresses $m=\left(\sigma_{11}+\sigma_{22}\right) a_{0}$ and the difference between stresses $m^{\prime}=\left(\sigma_{11}-\sigma_{22}\right) a_{0}$, multiplied by $a_{0}$. Therefore the stresses can be easily calculated from the $m$ and $m^{\prime}$ parameters, i.e.

$$
\sigma_{11}=\frac{m+m^{\prime}}{2 a_{0}} \quad \text { and } \quad \sigma_{22}=\frac{m-m^{\prime}}{2 a_{0}} .
$$

The uncertainty of the $a_{0}$ parameter is obtained directly from linear regression, while the uncertainties of the stresses can be calculated using the uncertainty propagation law. 
Two versions of the simple linear regression method can be applied in stress analysis. The first one, weighted simple linear regression (WSLR), is based on the minimization of the same merit function [equation (10)], which is done in two steps, i.e. separately for each of equations (16). When the straight lines are fitted to experimental points, explicit formulas for the $m, a_{0}$ and $m^{\prime}$ parameters and their statistical uncertainties can be derived [see Appendix A, equations (18)-(20)]. Although the new two-step WSLR procedure differs from the WLLS calculations (where $\sigma_{11}, \sigma_{22}$ and $a_{0}$ are adjusted simultaneously), the two methods should give similar results because in both cases the same merit function $\chi^{2}$ is minimized. In this work, another formulation of the merit function was also tested, in which equal weights were assigned to all measurement points, so the knowledge of individual weights was not necessary. In such a case, the solution can be easily obtained by substituting $w_{i}=1$ in equations (18)-(20). The latter approach, called ordinary simple linear regression (OSLR), is simpler because the experimental uncertainties are not needed in the calculation of the merit function. However, this method is not recommended because the low $2 \theta_{\{h k l\}}$ angle reflections have the same weights as those measured with large scattering angles.

\section{Sample characterization and experimental setup}

The first sample studied in the present paper is the Ni-base alloy with chemical composition given in Table 1, which has a
Table 1

Chemical composition (wt\%) of the Ni alloy (Inconel 690).

\begin{tabular}{llllllllll}
\hline $\mathrm{Ni}$ & $\mathrm{Cr}$ & $\mathrm{Fe}$ & $\mathrm{Si}$ & $\mathrm{Ti}$ & $\mathrm{Mn}$ & $\mathrm{C}$ & $\mathrm{Cu}$ & $\mathrm{P}$ & $\mathrm{S}$ \\
\hline Balance & 29.91 & 10.61 & 0.38 & 0.33 & 0.29 & 0.022 & 0.01 & 0.009 & 0.002 \\
\hline
\end{tabular}

Table 2

Single-crystal elastic constants $C_{i j}(\mathrm{GPa})$ and Zener factor $(A)$ for $\mathrm{Ni}$ crystals (Simmons \& Wang, 1971).

\begin{tabular}{llllllll}
\hline Material & $C_{11}$ & $C_{12}$ & $C_{13}$ & $C_{33}$ & $C_{44}$ & $C_{66}$ & $A$ \\
\hline $\mathrm{Ni}$ & 245 & 148 & 148 & 245 & 134 & 134 & 2.76 \\
\hline
\end{tabular}

relatively high elastic anisotropy of crystallites (Zener factor: 2.76; $c f$. Table 2). The sample surface was ground manually in one direction (under a load equal to $80 \mathrm{~N}$ and at a linear speed of $\left.88 \mathrm{~mm} \mathrm{~s}^{-1}\right)$. Such surface treatment results in a rough surface $\left(R_{\mathrm{a}}=3.3 \mu \mathrm{m}\right)$ consisting of ridges and furrows, oriented along the direction of grinding.

The second sample is an Ni coating electrodeposited on a $\mathrm{Cu}$ substrate (size equal to $15 \times 14 \times 1 \mathrm{~mm}$ ). A pure nickel plate anode was applied to the electrodeposition from an electrolyte composed of $300 \mathrm{~g} \mathrm{l}^{-1} \mathrm{NiSO}_{4} \cdot 6 \mathrm{H}_{2} \mathrm{O}$ and $60 \mathrm{~g} \mathrm{l}^{-1}$ $\mathrm{NiCl}_{2} \cdot 6 \mathrm{H}_{2} \mathrm{O}$. The bath temperature was maintained at $318 \pm$ $2 \mathrm{~K}$ and pH at $4 \pm 0.5$. The electrodeposition was carried out with current density equal to $5 \mathrm{~A} \mathrm{dm}^{-2}$. Before electrodeposition the substrate sample was polished using $\mathrm{SiC}$ paper
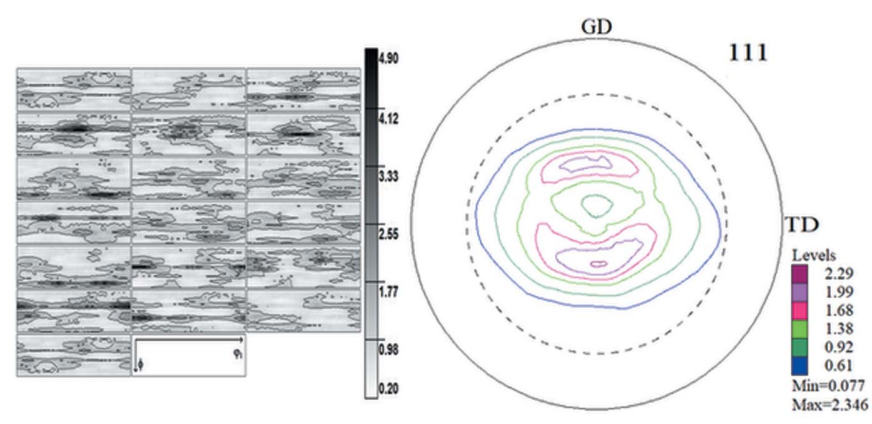

GD -

(a)

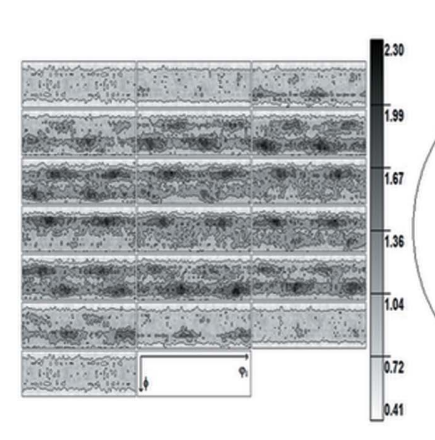

Figure 3 presented along the $\varphi_{2}$ axis and in the ranges $0 \leq \varphi_{1} \leq 360^{\circ}, 0^{\circ} \leq \Phi \leq 90^{\circ}$ (Bunge, 1993). 


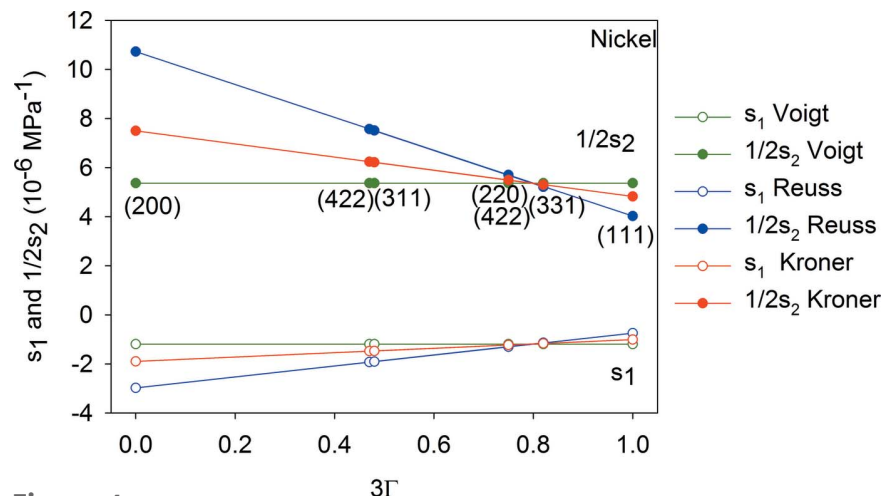

Figure 4

The $\frac{1}{2} s_{2}^{h k l}$ and $s_{1}^{h k l}$ constants as a function of $3 \Gamma$ calculated from the singlecrystal data (Table 2) for Ni, using Voigt (green lines), Eshelby-Kröner (red lines) and Reuss (blue lines) models.

with successively increased grit. The final polishing was done with 2500-grit paper. Then, the substrate was gently etched in $60 \%$ sulfuric acid. The average roughness of the deposited layer was $R_{\mathrm{a}}=0.45 \mu \mathrm{m}$.

To characterize crystallographic texture the $\{111\},\{200\}$, $\{220\}$ and $\{311\}$ incomplete pole figures were measured using $\mathrm{Cu} K \alpha$ radiation on a PANalytical X'Pert MRD diffractometer. In the case of the ground surface, the pole figures show almost orthorhombic sample symmetry, and a few significant maxima are seen on the determined ODF [Fig. 3(a)]. The electrodeposited Ni coating has a low and almost fibre-type texture [Fig. 3(b)].

The interplanar spacings used in the stress analysis were measured with $\mathrm{Cu} K \alpha \mathrm{X}$-ray radiation using the MGIXD method for the incident angle $\alpha=5^{\circ}$, corresponding to penetration depths $\tau=0.75$ and $0.9 \mu \mathrm{m}$ for the $\mathrm{Ni}$ alloy and electrodeposited Ni coating, respectively. The measurements were performed for $\phi=0^{\circ}$ and $\phi=90^{\circ}$ and for all available $h \mathrm{kl}$ reflections corresponding to different $\psi$ angles using a parallel-beam configuration. The incident beam optics comprised a Göbel mirror and Soller slit $\left(2.29^{\circ}\right)$ with a fixed divergence slit $\left(1 / 2^{\circ}\right)$, whereas the diffracted beam optics comprised a parallel plate collimator $\left(0.18^{\circ}\right)$ and Soller slit $\left(2.29^{\circ}\right)$.

\section{Results}

\subsection{Weighted least-squares method} used for data analysis

In the first approach, the WLLS fitting procedure was used and the $\langle a(\phi, \psi)\rangle_{\{h k l\}}$ lattice parameters, calculated from equation (9), were fitted to the experimental values. The calculations were based on minimizing the

Figure 5
Table 3

The values of stresses and $a_{0}$ parameter determined for two studied samples with different assumptions (incident angle $\alpha=5^{\circ}$ ), using the WLLS method.

The $\chi^{2}$ value corresponds to $\delta\left(2 \theta_{\{h k l\}}\right)=0.01^{\circ}$. Values in parentheses are the uncertainties on the least-significant digits.

\begin{tabular}{|c|c|c|c|c|c|c|}
\hline \multirow[b]{2}{*}{ Sample } & \multirow[b]{2}{*}{$\begin{array}{l}\text { Assumption } \\
\text { for XSFs }\end{array}$} & \multirow[b]{2}{*}{$\begin{array}{l}\text { Determined } \\
\text { quantities }\end{array}$} & \multicolumn{4}{|c|}{ Interaction model } \\
\hline & & & Reuss & $\begin{array}{l}\text { Free } \\
\text { surface }\end{array}$ & $\begin{array}{l}\text { Eshelby- } \\
\text { Kröner }\end{array}$ & Voigt \\
\hline \multirow{8}{*}{$\begin{array}{l}\text { Ground } \\
\mathrm{Ni} \text { alloy }\end{array}$} & \multirow{4}{*}{$\begin{array}{l}\text { ODF shown } \\
\text { in Fig. } 3(a)\end{array}$} & $\sigma_{11}(\mathrm{MPa})$ & $1042(51)$ & $1145(33)$ & $1269(35)$ & 1433(119) \\
\hline & & $\sigma_{22}(\mathrm{MPa})$ & $209(52)$ & $223(33)$ & $284(36)$ & $331(120)$ \\
\hline & & $a_{0}(\AA)$ & $3.5776(4)$ & $3.5774(3)$ & $3.5768(3)$ & $3.5762(8)$ \\
\hline & & $\chi^{2}$ & 21 & 8 & 7 & 62 \\
\hline & \multirow{4}{*}{$\begin{array}{l}\text { Quasi- } \\
\text { isotropic }\end{array}$} & $\sigma_{11}(\mathrm{MPa})$ & 1056(49) & 1141(31) & 1277(40) & 1437(119) \\
\hline & & $\sigma_{22}(\mathrm{MPa})$ & 209(49) & $203(30)$ & $278(40)$ & $324(117)$ \\
\hline & & $a_{0}(\AA)$ & $3.5776(4)$ & $3.5774(2)$ & $3.5768(3)$ & $3.5762(8)$ \\
\hline & & $\chi^{2}$ & 19 & 7 & 9 & 62 \\
\hline \multirow{8}{*}{$\begin{array}{l}\text { Deposited } \\
\mathrm{Ni}\end{array}$} & \multirow{4}{*}{$\begin{array}{l}\text { ODF shown } \\
\text { in Fig. } 3(b)\end{array}$} & $\sigma_{11}(\mathrm{MPa})$ & $525(30)$ & $546(31)$ & $593(36)$ & $618(61)$ \\
\hline & & $\sigma_{22}(\mathrm{MPa})$ & $530(30)$ & $550(31)$ & $597(35)$ & 621(61) \\
\hline & & $a_{0}(\AA)$ & $3.5232(2)$ & $3.5232(2)$ & $3.5227(2)$ & $3.5225(4)$ \\
\hline & & $\chi^{2}$ & 4 & 4 & 5 & 12 \\
\hline & \multirow{4}{*}{$\begin{array}{l}\text { Quasi- } \\
\text { isotropic }\end{array}$} & $\sigma_{11}(\mathrm{MPa})$ & $511(29)$ & $533(30)$ & $577(35)$ & $602(59)$ \\
\hline & & $\sigma_{22}(\mathrm{MPa})$ & $516(29)$ & $537(30)$ & $580(35)$ & $606(59)$ \\
\hline & & $a_{0}(\mathrm{~A})$ & $3.5232(2)$ & $3.5232(2)$ & $3.5228(2)$ & $3.5226(4)$ \\
\hline & & $\chi^{2}$ & 4 & 4 & 5 & 12 \\
\hline
\end{tabular}

merit function $\chi^{2}$ [equation (10)]. The $F_{i j}(h k l, \phi, \psi, f)$ values used in stress analysis were calculated from the SECs given in Table 2, assuming random grain orientations. The calculations were then repeated taking into account the ODFs shown in
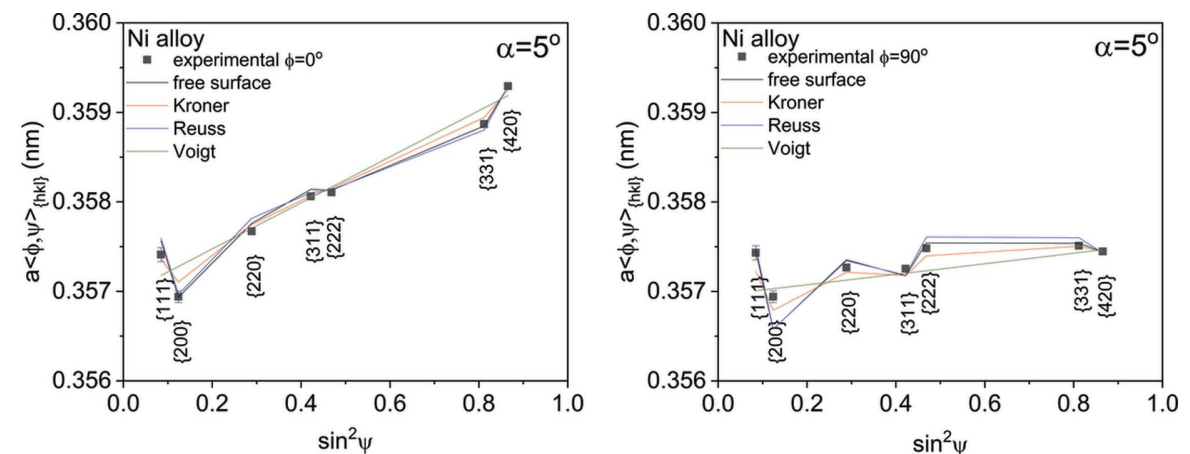

(a)
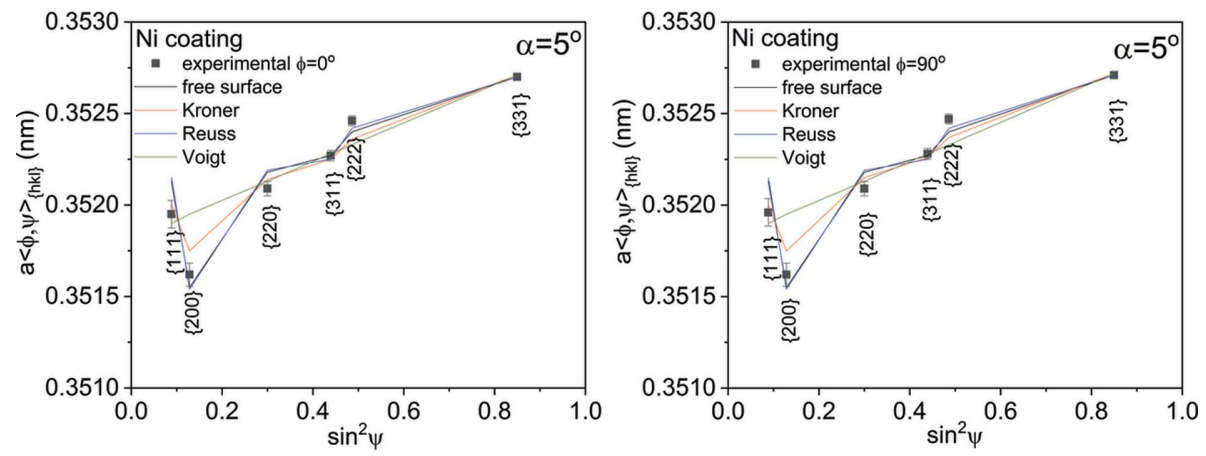

(b)

Examples of $\langle a(\phi, \psi)\rangle_{\{h k l\}}$ versus $\sin ^{2} \psi$ plots for the incident angle $\alpha=5^{\circ}$, compared for different grain interaction models. The results for the ground sample $(a)$ and electrodeposited layer $(b)$ are shown. Error bars correspond to $\delta\left(2 \theta_{\{h k l\}}\right)=0.01^{\circ}$. 

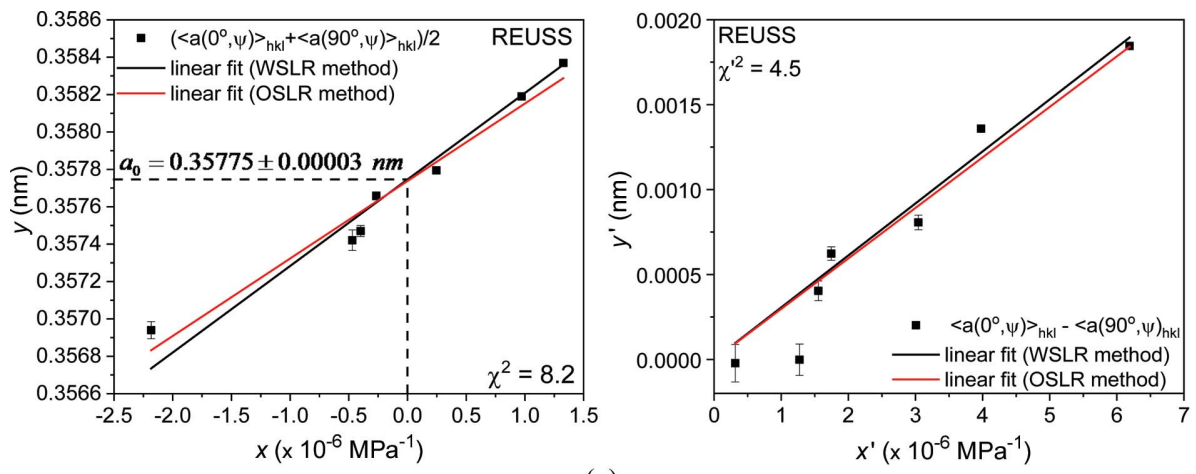

(a)
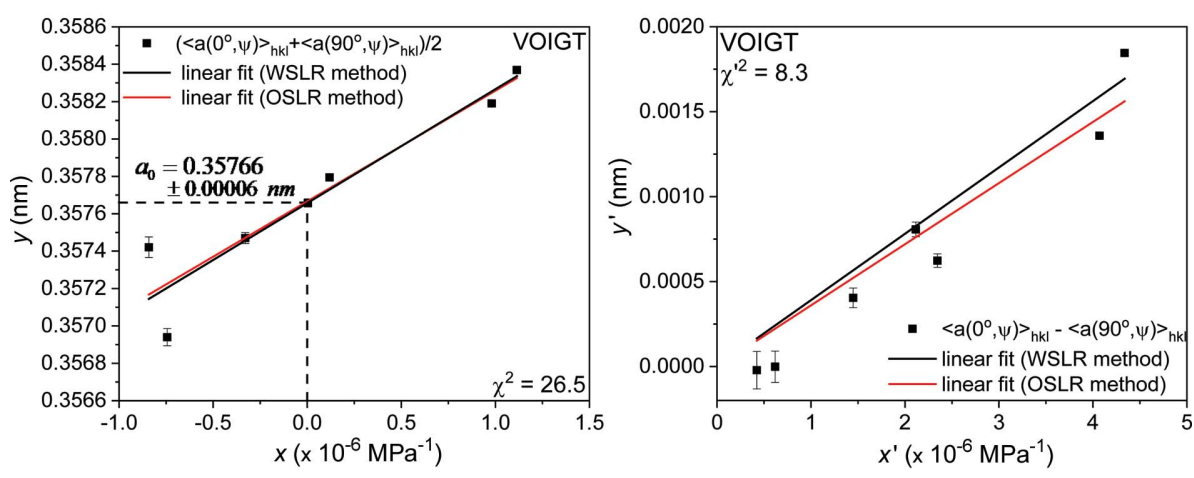

(b)
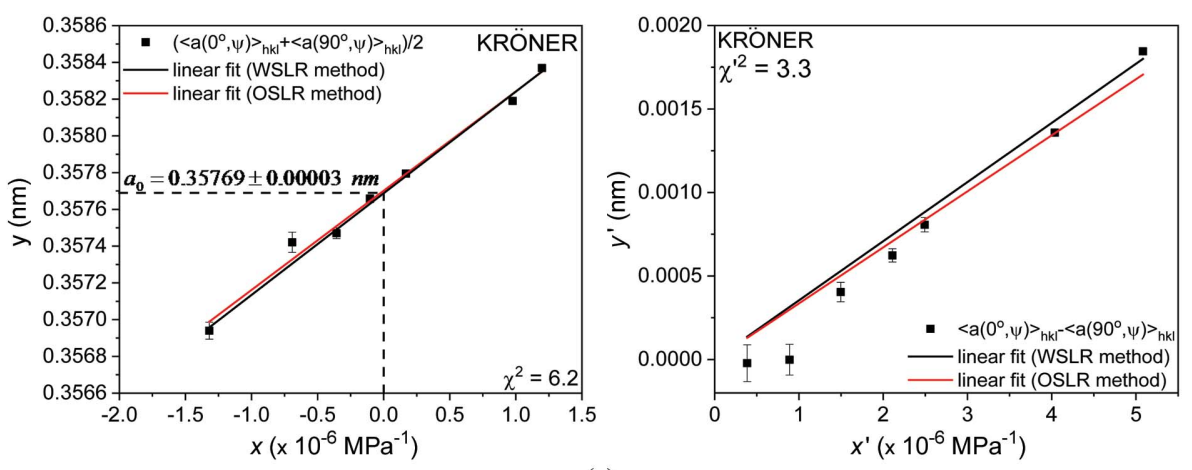

(c)
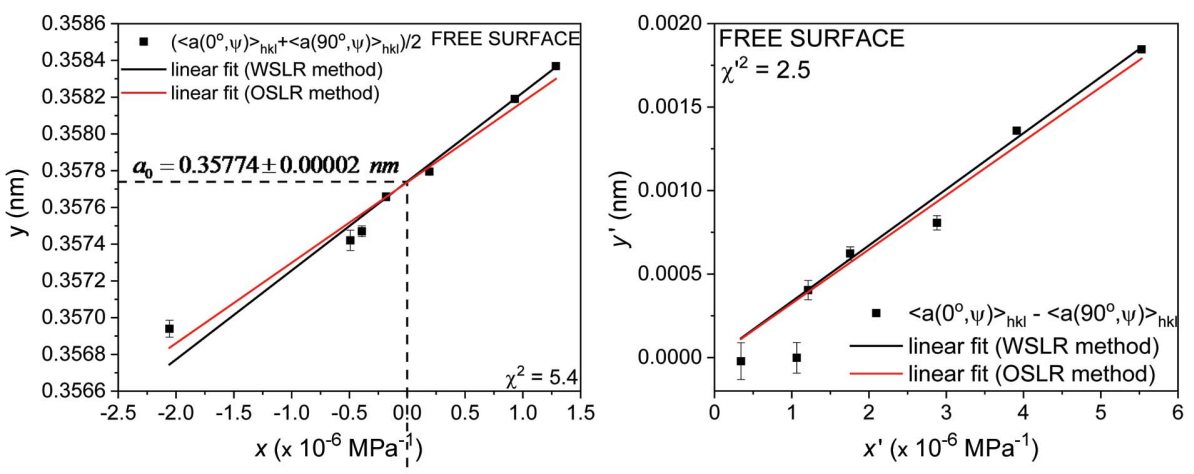

$(d)$

Figure 6

Simple linear regression results for the $y=m x+a_{0}$ and $y^{\prime}=m^{\prime} x^{\prime}$ functions, calculated using the WSLR and OSLR methods. Analysis of the MGIXD data $\left(\alpha=5^{\circ}\right)$ obtained for the ground Ni alloy was performed. Four different grains interaction models with the assumption of quasi-isotropic material were used to calculate XSFs: $(a)$ Reuss, $(b)$ Voigt, $(c)$ Eshelby-Kröner and $(d)$ free-surface models. The $y$ intercepts equal to the $a_{0}$ value and $\chi^{2}$ are given for the WSLR method. Error bars correspond to $\delta\left(2 \theta_{\{h k l\}}\right)=0.01^{\circ}$.
Fig. 3. Four theoretical models of grain interaction were applied, i.e. Reuss, Voigt, Eshelby-Kröner and free-surface methods. The dependences of the XECs $\frac{1}{2} s_{2}^{h k l}$ and $s_{1}^{h k l}$ versus orientation parameter $3 \Gamma=3\left(h^{2} k^{2}+h^{2} l^{2}+k^{2} l^{2}\right) /\left(h^{2}+\right.$ $\left.k^{2}+l^{2}\right)^{2}$ for a quasi-isotropic sample are presented in Fig. 4. In this figure the results for all interaction models are shown, except for the free-surface model. In the case of the latter approach the $s_{2}^{h k l}$ and $s_{1}^{h k l}$ constants cannot be defined because the grain interaction depends on the direction with respect to the sample. The results of the Reuss and Eshelby-Kröner models confirm a marked elastic anisotropy of the calculated XECs, caused by elastic anisotropy of the Ni crystal.

The residual stresses (biaxial state, assuming $\sigma_{33}=0$ ) and stress-free parameter $a_{0}$ determined for the two studied samples using different interaction models are shown in Table 3. In this table the results of calculations, taking into account measured textures (Fig. 3), are compared with those for which the quasi-isotropic XECs were assumed.

Analysing the values of stresses and lattice parameter $a_{0}$ calculated using different interaction models (Table 3 ) it can be concluded that the results obtained taking into account crystallographic texture in calculations of XSFs are almost equal to these calculated with the assumption of a quasiisotropic sample, within the uncertainty range. Because the effect of texture is not significant in either sample, in further calculations of XSFs only the quasi-isotropic approach is considered.

The experimental $\langle a(\phi, \psi)\rangle_{\{h k l\}}$ versus $\sin ^{2} \psi$ plots, obtained for both investigated samples with incident angle $\alpha=5^{\circ}$ and assuming quasi-isotropic XECs, are shown in Fig. 5. The substantial nonlinearities of these plots are certainly related to the significant anisotropy of the SECs. In this figure one can see that the trends of nonlinearity are replicated by the lines obtained with the EshelbyKröner, Reuss and free-surface models. It is not possible to visually determine which model is closest to the experimental results. The values of parameter $\chi^{2}$ calculated for both studied samples (Table 3) show the best accordance with 
experiment for the Eshelby-Kröner and free-surface models, and slightly lower agreement for the Reuss model. Only in the case of the Voigt model are the experimental points far away from the predicted linear $\langle a(\phi, \psi)\rangle_{\{h k l\}}$ versus $\sin ^{2} \psi$ plot, and the $\chi^{2}$ value is significantly higher, i.e. this model does not correctly take into account the elastic anisotropy of the studied Ni samples.

\subsection{Linear regression methods for data analysis}

In the second approach, simple linear regression fittings on the basis of equation (16) were done and the values $a_{0}, \sigma_{11}$ and $\sigma_{22}$ were determined [equation (17)], as described in Section 2.2.2. Calculations were performed with two assumptions concerning weights of the measured points, i.e. the WSLR and OSLR procedures were applied. Four different grain interaction models were used in the calculations. The $y$ versus $x$ and $y^{\prime}$ versus $x^{\prime}$ plots $\left(\alpha=5^{\circ}\right)$ are presented in Fig. 6 for the ground sample and in Fig. 7 for the deposited coating. The intercept of the fitted $y$ versus $x$ linear function with the $y$ axis (see the left hand side plots in Figs. 6 and 7) gives the $a_{0}$ value. The slopes of the two fitted lines determine the values of $\sigma_{11}$ and $\sigma_{22}$, calculated using equation (17). Note that only one parameter, $m^{\prime}$, is fitted in the case of the $y^{\prime}$ versus $x^{\prime}$ function, and the resulting straight line passes through the beginning of the coordinate system. In the case of a ground sample, an apparent discrepancy of the fitted line with experimental points is seen for small values of $x^{\prime}$ (see the right hand side plots in Fig. 6 and Table 4). It is seen that different straight lines were fitted using the WSLR and OSLR methods owing to the difference in weighting of the experimental data. The final results of stress and lattice parameter analysis obtained using different grain interaction models and different adjustment methods (WLLS, WSLR and OSLR) are summarized in Fig. 8 in the form of histograms.

\section{Discussion}

Tensile stresses found in the ground $\mathrm{Ni}$ alloy sample were generated as a result of the temperature gradient during surface processing (Table 3 and Fig. 8). The residual stresses in the direction of grinding $\left(\sigma_{11}\right)$ are large, while for the perpendicular direction $\left(\sigma_{22}\right)$ they are
Figure 7

(d)

The same comparison as in Fig. 6 but for the electrodeposited Ni coating. 
Table 4

The $\chi^{2}$ and $\chi^{\prime 2}$ values obtained using the WSLR and OSLR methods with XECs calculated using different models [assuming $\delta\left(2 \theta_{\{h k l\}}\right)=0.01^{\circ}$ ].

\begin{tabular}{llll}
\hline Sample & $\begin{array}{l}\text { Model for XEC } \\
\text { calculations }\end{array}$ & $\chi^{2}$ for $y=m x+a_{0}$ & $\chi^{\prime 2}$ for $y^{\prime}=m^{\prime} x^{\prime}$ \\
\hline \multirow{2}{*}{ Ground Ni } & Voigt & $\chi^{2}=26.5$ & $\chi^{\prime 2}=8.3$ \\
& Eshelby-Kröner & $\chi^{2}=6.2$ & $\chi^{\prime 2}=3.3$ \\
& Free surface & $\chi^{2}=5.4$ & $\chi^{\prime 2}=2.5$ \\
& Reuss & $\chi^{2}=8.2$ & $\chi^{\prime 2}=4.5$ \\
Ni coating & Voigt & $\chi^{2}=18.6$ & $\chi^{\prime 2}=0.13$ \\
& Eshelby-Kröner & $\chi^{2}=7.4$ & $\chi^{\prime 2}=0.13$ \\
& Free surface & $\chi^{2}=6.1$ & $\chi^{\prime 2}=0.13$ \\
& Reuss & $\chi^{2}=6.2$ & $\chi^{\prime 2}=0.12$ \\
\hline
\end{tabular}

much smaller. This effect can be explained as due to the surface topography (ridges and furrows) as well as the nature of one-directional processing of the sample surface. In the case of the electrodeposited $\mathrm{Ni}$ coating, similar values of tensile stresses were found in two perpendicular directions $\left(\sigma_{11} \simeq\right.$ $\sigma_{22}$ ), which is explained by the axial symmetry of the deposition process. Therefore the proposed methods of data treatment were tested on two different states of the surface stress, i.e. $\sigma_{11} \gg \sigma_{22}$ and $\sigma_{11} \simeq \sigma_{22}$.

Three different procedures of stress analysis were applied to treat the data obtained using MGIXD geometry. It was found that all the tested adjustment methods give similar values of $\sigma_{11}, \sigma_{22}$ and $a_{0}$, as well as uncertainties of their determination. The results of the weighted methods (WSLR and WLLS) are almost identical, because in both cases the same merit function was minimized. A significant elastic anisotropy of the XECs (or XSFs) is seen on the $\left\langle a(\phi, \psi)_{\{h k l\}}\right.$ versus $\sin ^{2} \psi$ plots obtained using the WLLS method, where the equivalent lattice parameter is far from the linear function for the 200 and 111 reflections. In fact, the strongest elastic anisotropy occurs for the corresponding crystal directions in the case of Ni crystals ( $c f$. Fig. 4). It was also found that the influence of texture on the calculated XSFs and consequently stresses calculated by the WLLS method was not significant for the studied samples. Therefore, simplified WSLR and OSLR methods can be used to analyse experimental data, with XECs calculated for a quasi-isotropic sample (with random grain orientations).

The advantage of the new analysis approaches proposed in this paper (i.e. the WSLR and OSLR procedures) is that the considered plots, given by equations (16), are linear, while elastic anisotropy is taken into account in the calculations. Therefore, the departure of the experimental points from a straight line can be easily noticed (Figs. 6 and 7). However, as was proven above, the disagreement between experiment and the fitted line is not caused by shear stresses. The reasons for the observed discrepancies must be discussed.

We emphasize that in the case of the $y=m x+a_{0}$ plot [where the sum of the lattice parameters measured for $\phi=0^{\circ}$ and $\phi=$ $90^{\circ}$ is considered in equations $(14 a)$ and $\left.(15 a)\right]$ the slope of the fitted line is proportional to the sum of plane stresses $\left(\sigma_{11}+\right.$ $\sigma_{22}$ ), while the departure of experimental points from the fitted line informs us about accumulated errors caused by instrumental errors, inaccuracies of the XECs used in the analysis (for example due to texture which is not taken into account), microstresses present in the sample, stress
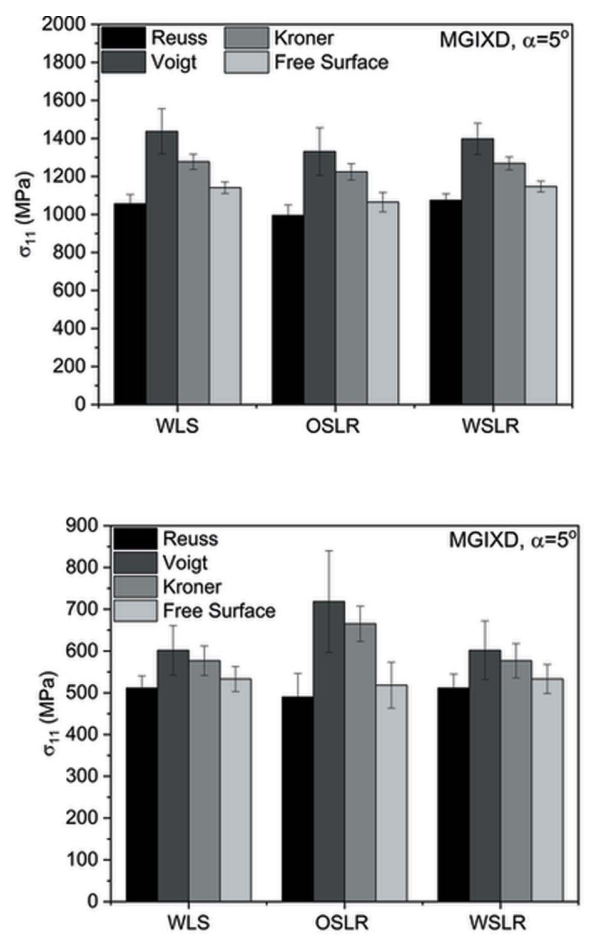

Figure 8

Comparison of the results obtained using four models of grain interaction and three analysis methods. The values of biaxial stresses and stress-free lattice constant obtained using the MGIXD method with $\alpha=5^{\circ}$ for the ground Ni alloy sample $(a)$ and deposited Ni layer $(b)$ are presented.

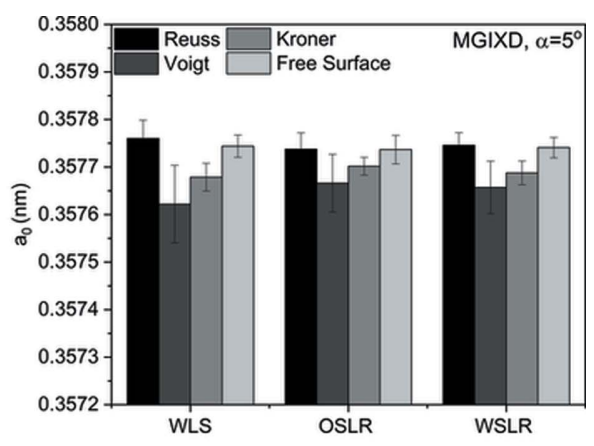

(a)
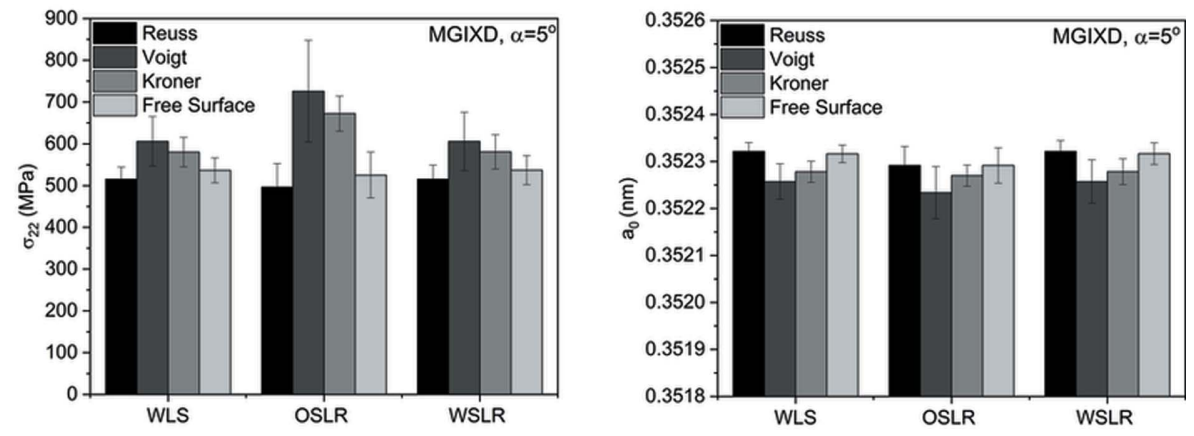

(b) 
heterogeneity etc. On the other hand, the slope of the fitted $y^{\prime}=m^{\prime} x^{\prime}$ line and its accordance with the difference in the lattice parameters measured for the two $\phi$ directions [ $c f$. equations $(14 b)$ and $(15 b)]$ verifies the axial symmetry of the sample with respect to the direction normal to the sample surface. For axial symmetry, i.e. when $\sigma_{11}=\sigma_{22}$, the $y^{\prime}=m^{\prime} x^{\prime}$ line should be horizontal. Furthermore, possible instrumental and some other systematic errors can subtract from and reduce each other when the difference in lattice parameters is calculated for the two sample orientations.

Undoubtedly, axial symmetry is observed in the case of the deposited Ni layer investigated in the present research. The fitted line $y^{\prime}=m^{\prime} x^{\prime}$ perfectly passes through the experimental points, causing the uncertainty bars to be overestimated because of possible compensation of some systematic errors (see also the small values of $\chi^{\prime 2}$ shown in Table 4). The axial symmetry of the deposited $\mathrm{Ni}$ coating is also confirmed by two other results, i.e. the approximated fibre texture shown in Fig. 3(b) and $\sigma_{11} \simeq \sigma_{22}$ (Table 3 and Fig. 8). It is also clear that the ground sample does not show axial symmetry, as can be seen in the pole figures presented in Fig. 3(a). Moreover, in this case different stresses were determined for the two measured directions (i.e. $\sigma_{11} \gg$ $\sigma_{22}$ ). The lack of axial symmetry and the difference in principal stresses result in a significant slope of the $y^{\prime}=m^{\prime} x^{\prime}$ lines and a noticeable departure of the experimental data from these lines, as seen in Figs. 6 and 7. A significant disagreement was found for small values of $x^{\prime}$, i.e. for the points with the largest error bar values, corresponding to the reflections having the lowest multiplicity (i.e. 111 and 200 reflections) and measured using the lowest values of the scattering angle. These reflections are the most sensitive to sample anisotropy, i.e. effects of texture and anisotropic microstresses (Hauk, 1997).

In spite of the simplifications assumed in the proposed new data treatments (i.e. WSLR and OSLR), the results obtained for both investigated samples are reasonable (experimental points are close to fitted straight lines) and they are confirmed by the weighted linear least-squares method (WLLS), which has been well tested in other work (see Fig. 8). For the considered grain interaction models such as Reuss, EshelbyKröner and free-surface, the theoretical results correctly fit to the experimental points but it is not possible to distinguish which model deals better with the elastic anisotropy ( $c f$. Tables 3 and 4). The worst result was always obtained with the Voigt model, in which the XECs do not depend on the $h k l$ reflection (Fig. 4). This in turn leads to a large spread of the experimental points around the fitted lines (for all types of analysis performed in this work). From our previous investigations of other materials (Marciszko et al., 2015, 2016), in which external stress was applied to the sample, the best agreement of experimental and theoretical XSFs was obtained for the Reuss and free-surface models when the measurements were done using MGIXD for shallow penetration depths.
Finally, to check the potential influence of the shear stresses on the obtained results, the extension of the WSLR and OSLR methods is proposed using the linear functions given by equations (24) and (25), which are derived in Appendix $B$. These functions were fitted by straight lines using the WSLR method with free-surface XECs, and the results for both studied samples are shown in Fig. 9. Insignificant values of shear stresses $\sigma_{13}$ and $\sigma_{23}$ were found in both samples, and therefore the departure of the experimental points from the theoretical lines in Figs. 6 and 7 is not caused by these stresses.

\section{Conclusions}

It can be concluded that the proposed new methods of MGIXD data treatment were successfully tested on $\mathrm{Ni}$ samples exhibiting significant elastic anisotropy of crystals. An important advantage of the proposed analysis is its simplicity, resulting from linear regression in which the straight line is fitted to experimental data, while taking into account the elastic anisotropy of the studied material. The presented comparison of the measured points with the straight line allows straightforward interpretation, demonstrating if the assumptions concerning grain interactions, texture and shear stresses are correct. Moreover, the value of the stress-free lattice parameter $a_{0}$ can be directly determined as the intercept of the fitted line with the $y$ axis. All necessary equations used in the analysis are provided in this paper, including Appendices $A$ and $B$.

It was found that the three tested methods of adjustment procedure (i.e. WLLS, WSLR and OSLR) give similar values and uncertainties of the determined stresses and stress-free 
lattice parameter. The anisotropy of the XSFs (or XECs) is well predicted by the Reuss, Eshelby-Kröner and free-surface methods, but it is not possible to decide which model is the best one. The worst result was always obtained with the Voigt model in which the XSF (or XEC) values do not depend on the $h k l$ reflection.

\section{APPENDIX $A$}

The explicit formulas for the weighted solution of the linear least-squares method (WSLR) can be derived for the functions given in equation (16). The two parameters $\left(m, a_{0}\right)$ of the first linear function and their statistical uncertainties $\left[u(m), u\left(a_{0}\right)\right]$ are given by

$$
\begin{aligned}
& m=\frac{\sum_{n=1}^{N} w_{n} \sum_{n=1}^{N} w_{n} x_{n} y_{n}-\sum_{n=1}^{N} w_{n} x_{n} \sum_{n=1}^{N} w_{n} y_{n}}{D}, \\
& \left.u(m)=\chi^{2} \frac{\sum_{n=1}^{N} w_{n}}{D}\right)^{1 / 2}, \\
& a_{0}=\frac{\sum_{n=1}^{N} w_{n} y_{n} \sum_{n=1}^{N} w_{n} x_{n}^{2}-\sum_{n=1}^{N} w_{n} x_{n} \sum_{n=1}^{N} w_{n} x_{n} y_{n}}{D}, \\
& u\left(a_{0}\right)=\left(\chi^{2} \frac{\sum_{n=1}^{N} w_{n} x_{n}^{2}}{D}\right)^{1 / 2},
\end{aligned}
$$

where $\chi^{2}=[1(N-2)] \sum_{n=1}^{N} w_{n}\left(y_{n}-m x_{n}-a_{0}\right)^{2}, w_{n}=1 / \delta^{2}\left(y_{n}\right)$ and $D=\sum_{n=1}^{N} w_{n} \sum_{n=1}^{N} w_{n} x_{n}^{2}-\left(\sum_{n=1}^{N} w_{n} x_{n}\right)^{2}$.

The parameter $m^{\prime}$ of the second linear function and its A-type uncertainty $u\left(m^{\prime}\right)$ can be determined from

$$
m^{\prime}=\frac{\sum_{n=1}^{N} w_{n}^{\prime} x_{n}^{\prime} y_{n}^{\prime}}{D^{\prime}}, \quad u\left(m^{\prime}\right)=\left(\frac{\chi^{\prime 2}}{D^{\prime}}\right)^{1 / 2}
$$

where $\chi^{\prime 2}=[1 /(N-1)] \sum_{n=1}^{N} w_{n}^{\prime}\left(y_{n}^{\prime}-m^{\prime} x_{n}^{\prime}\right)^{2}, w_{n}^{\prime}=1 / \delta^{2}\left(y_{n}^{\prime}\right)$ and $D^{\prime}=\sum_{n=1}^{N} w_{n}^{\prime} x_{n}^{\prime 2}$.

Note that the solutions of the OSLR method can be easily obtained by substituting $w_{n}=w_{n}^{\prime}=1$ in equations (18)-(20).

\section{APPENDIX $B$}

Assuming fibre texture or a quasi-isotropic sample, equations similar to equation (12) can be written as

$$
\begin{aligned}
F_{13}\left(h k l, \phi=0^{\circ}, \psi, f\right) & =F_{23}\left(h k l, \phi=90^{\circ}, \psi, f\right) \\
& =M(h k l, \psi), \\
F_{23}\left(h k l, \phi=0^{\circ}, \psi, f\right) & =F_{13}\left(h k l, \phi=90^{\circ}, \psi, f\right) \\
& =N(h k l, \psi),
\end{aligned}
$$

where $M(h k l, \psi)$ and $N(h k l, \psi)$ depend on the $h k l$ reflection and $\psi$ angle but not on the $\phi$ angle.

Then equations (13) can be written with additional terms depending on shear stresses $\sigma_{13}$ and $\sigma_{23}$ :

$$
\begin{aligned}
\left\langle a\left(\phi=0^{\circ}, \psi\right)\right\rangle_{\{h k l\}}= & {\left[G(h k l, \psi) \sigma_{11}+H(h k l, \psi) \sigma_{22}\right.} \\
& \left.+M(h k l, \psi) \sigma_{13}+N(h k l, \psi) \sigma_{23}\right] a_{0}+a_{0}, \\
\left\langle a\left(\phi=90^{\circ}, \psi\right)\right\rangle_{\{h k l\}}= & {\left[H(h k l, \psi) \sigma_{11}+G(h k l, \psi) \sigma_{22}\right.} \\
& \left.+N(h k l, \psi) \sigma_{13}+M(h k l, \psi) \sigma_{23}\right] a_{0}+a_{0} .
\end{aligned}
$$

When $m, m^{\prime}$ and $a_{0}$ [equations (16)] are determined from the least-squares procedure described in Section 2.2.2, the deviations from linearity for the sum and difference of the above equations can be expressed by

$$
\begin{aligned}
& \frac{\left\langle a\left(\phi=0^{\circ}, \psi\right)\right\rangle_{\{h k l\}}+\left\langle a\left(\phi=90^{\circ}, \psi\right)\right\rangle_{\{h k l\}}}{2}-\left(m x+a_{0}\right) \\
& =\frac{M(h k l, \psi)+N(h k l, \psi)}{2}\left(\sigma_{13}+\sigma_{23}\right) a_{0}, \\
& \left\langle a\left(\phi=0^{\circ}, \psi\right)\right\rangle_{\{h k l\}}-\left\langle a\left(\phi=90^{\circ}, \psi\right)\right\rangle_{\{h k l\}}-m^{\prime} x^{\prime} \\
& =[M(h k l, \psi)-N(h k l, \psi)]\left(\sigma_{13}-\sigma_{23}\right) a_{0},
\end{aligned}
$$

or in the case of a quasi-isotropic sample by

$$
\begin{aligned}
& \frac{\left\langle a\left(\phi=0^{\circ}, \psi\right)\right\rangle_{\{h k l\}}+\left\langle a\left(\phi=90^{\circ}, \psi\right)\right\rangle_{\{h k l\}}}{2}-\left(m x+a_{0}\right) \\
& =\left(\frac{1}{4} s_{2}^{h k l} \sin 2 \psi\right)\left(\sigma_{13}+\sigma_{23}\right) a_{0}, \\
& \left\langle a\left(\phi=0^{\circ}, \psi\right)\right\rangle_{\{h k l\}}-\left\langle a\left(\phi=90^{\circ}, \psi\right)\right\rangle_{\{h k l\}}-m^{\prime} x^{\prime} \\
& =\left(\frac{1}{2} s_{2}^{h k l} \sin 2 \psi\right)\left(\sigma_{13}-\sigma_{23}\right) a_{0} .
\end{aligned}
$$

Both sets of equations (23) and (24) can be rewritten in the linear form

$$
w=n z, \quad w^{\prime}=n^{\prime} z^{\prime},
$$

where $w=\left[\left\langle a\left(\phi=0^{\circ}, \psi\right)\right\rangle_{\{h k l\}}+\left\langle a\left(\phi=90^{\circ}, \psi\right)\right\rangle_{\{h k l\}}\right] / 2-$ $\left(m x+a_{0}\right), z=[M(h k l, \psi)+N(h k l, \psi)] / 2, n=\left(\sigma_{13}+\sigma_{23}\right) a_{0}$, $w^{\prime}=\left\langle a\left(\phi=0^{\circ}, \psi\right)\right\rangle_{\{h k l\}}-\left\langle a\left(\phi=90^{\circ}, \psi\right)\right\rangle_{\{h k l\}}-m^{\prime} x^{\prime}$ and $z^{\prime}=$ $[M(h k l, \psi)-N(h k l, \psi)]$. For a quasi-isotropic sample $z=\frac{1}{4} s_{2}^{h k l} \sin 2 \psi, z^{\prime}=\frac{1}{2} s_{2}^{h k l} \sin 2 \psi$ and $m^{\prime}=\left(\sigma_{13}-\sigma_{23}\right) a_{0}$.

In order to determine the values of the $\sigma_{13}$ and $\sigma_{23}$ shear stresses, the WSLR and OSLR methods can be applied for equations (25), analogously to what was previously done in the case of equations (16).

\section{Funding information}

This work was partially supported by grants from the National Science Centre, Poland (NCN), Nos. UMO-2017/25/B/ST8/ 00134 and UMO-2014/15/D/ST8/00542.

\section{References}

Baczmański, A., Braham, C. \& Seiler, W. (2003). Philos. Mag. 83, 3225-3246.

Baczmanski, A., Lipinski, P., Tidu, A., Wierzbanowski, K. \& Pathiraj, B. (2008). J. Appl. Cryst. 41, 854-867.

Baczmański, A., Wierzbanowski, K., Haije, W. G., Helmholdt, R. B., Ekambaranathan, G. \& Pathiraj, B. (1993). Cryst. Res. Technol. 28, 229-243.

Barral, M., Lebrun, J., Sprauel, J. \& Maeder, G. (1987). Metall. Trans. A, 18, 1229-1238.

Brakman, C. M. (1987). Philos. Mag. A, 55, 39-58. 
Bunge, H.-J. (1993). Texture Analysis in Materials Science: Mathematical Methods, 1st ed. Göttingen: Cuvillier.

Dölle, H. (1979). J. Appl. Cryst. 12, 489-501.

Dölle, H. \& Cohen, J. B. (1980). Metall. Trans. A, 11, 159-164.

Dölle, H. \& Hauk, V. (1976). Haerterei-Techn. Mitt. 31, 165-168.

Eshelby, J. D. (1957). Proc. R. Soc. London Ser. A, 241, 376-396.

Genzel, C. (1999). J. Appl. Cryst. 32, 770-778.

Hauk, V. (1997). Structural and Residual Stress Analysis by Nondestructive Methods. Amsterdam: Elsevier.

Kröner, E. (1961). Acta Metall. 9, 155-161.

Leeuwen, M. van, Kamminga, J.-D. \& Mittemeijer, E. J. (1999). J. Appl. Phys. 86, 1904-1914.

Leoni, M., Welzel, U., Lamparter, P., Mittemeijer, E. J. \& Kamminga, J.-D. (2001). Philos. Mag. A, 81, 597-623.

Marciszko, M., Baczmański, A., Braham, C., Wróbel, M., Seiler, W., Wroński, S. \& Berent, K. (2016). J. Appl. Cryst. 49, 85-102.

Marciszko, M., Baczmański, A., Wierzbanowski, K., Wróbel, M., Braham, C., Chopart, J.-P., Lodini, A., Bonarski, J., Tarkowski, L. \& Zazi, N. (2012). Appl. Surf. Sci. 266, 256-267.

Marciszko, M., Baczmański, A., Wróbel, M., Seiler, W., Braham, C., Donges, J., Śniechowski, M. \& Wierzbanowski, K. (2013). Thin Solid Films, 530, 81-84.

Marciszko, M., Baczmański, A., Wróbel, M., Seiler, W., Braham, C., Wroński, S. \& Wawszczak, R. (2015). J. Appl. Cryst. 48, 492-509.
Matthies, S., Humbert, M. \& Schuman, C. (1994). Phys. Status Solidi $B, \mathbf{1 8 6}, \mathrm{K} 41-\mathrm{K} 44$.

Noyan, I. C. \& Cohen, J. B. (1987). Residual Stress: Measurement by Diffraction and Interpretation. New York: Springer.

Press, W. H., Flannery, B. P., Teukolsky, S. A. \& Vetterling, W. T. (1992). Numerical Recipes in C: the Art of Scientific Computing, 2nd ed. New York: Cambridge University Press.

Reuss, A. (1929). Z. Angew. Math. Mech. 9, 49-58.

Simmons, G. \& Wang, H. (1971). Single Crystal Elastic Constants and Calculated Aggregate Properties, 2nd ed. Cambridge, London: MIT Press.

Skrzypek, S. J., Baczmański, A., Ratuszek, W. \& Kusior, E. (2001). J. Appl. Cryst. 34, 427-435.

Thompson, P., Cox, D. E. \& Hastings, J. B. (1987). J. Appl. Cryst. 20, 79-83.

Van Acker, K., De Buyser, L., Celis, J. P. \& Van Houtte, P. (1994). J. Appl. Cryst. 27, 56-66.

Van Houtte, P. \& De Buyser, L. (1993). Acta Metall. Mater. 41, 323 336.

Voigt, W. (1928). Lehrbuch der Kristallphysik. Leipzig: Teubner.

Vook, R. W. \& Witt, F. (1965). J. Appl. Phys. 36, 2169-2171.

Welzel, U., Ligot, J., Lamparter, P., Vermeulen, A. C. \& Mittemeijer, E. J. (2005). J. Appl. Cryst. 38, 1-29.

Welzel, U. \& Mittemeijer, E. J. (2003). J. Appl. Phys. 93, 9001-9011. 